\title{
At First Glance: Psoriatic Response to Transdermal Nicotine Patch Application
}

\author{
Joseph M. LaRochelle ${ }^{a, b} \quad$ Victoria R. Dimitriades ${ }^{c}$ \\ ${ }^{a}$ College of Pharmacy, Xavier University of Louisiana, New Orleans, LA, USA; ${ }^{\text {bSchool }}$ \\ of Medicine, Louisiana State University Health Sciences Center, New Orleans, LA, USA; \\ 'Division of Pediatric Allergy, Immunology and Rheumatology, University of California Davis \\ Health, Sacramento, CA, USA
}

\section{Keywords}

Contact dermatitis $\cdot$ Psoriasis $\cdot$ Rash $\cdot$ Nicotine

\begin{abstract}
Contact dermatitis and psoriasis are common skin disorders which represent two distinct pathologies. Skin disorders heavily rely on corresponding history for diagnosis; this case demonstrates the challenges of relying on history alone for final identification. A patient presented to clinic for evaluation of a new rash on his abdomen. Past medical history was notable for recent initiation of a smoking cessation program utilizing nicotine patches as well as a pharyngitis treated with antibiotics 1 week prior. Despite use of topical steroid and cessation of the patches, the well-demarcated rash became more generalized. Patch testing for the nicotine patch and chemical sensitizers was negative and an eventual biopsy was consistent with guttate psoriasis. Contact dermatitis relies heavily on clinical history for diagnosis. This case demonstrates that history can be misleading. In retrospect, it is likely that a preceding presumed streptococcal infection was the primary inducer of guttate psoriasis.
\end{abstract}

(C) 2020 The Author(s)

Published by S. Karger AG, Basel

\section{Case Report}

Contact dermatitis (CD) and psoriasis are skin disorders which represent two distinct pathologies. Although both are initiated by keratinocytes and T cells, the former is mediated by a delayed-type IV hypersensitivity reaction and the latter is mediated predominantly by proinflammatory cytokines released by Th1 and Th17 cells [1]. Skin disorders rely on corre- 
Fig. 1. Application point of transdermal nicotine patch.

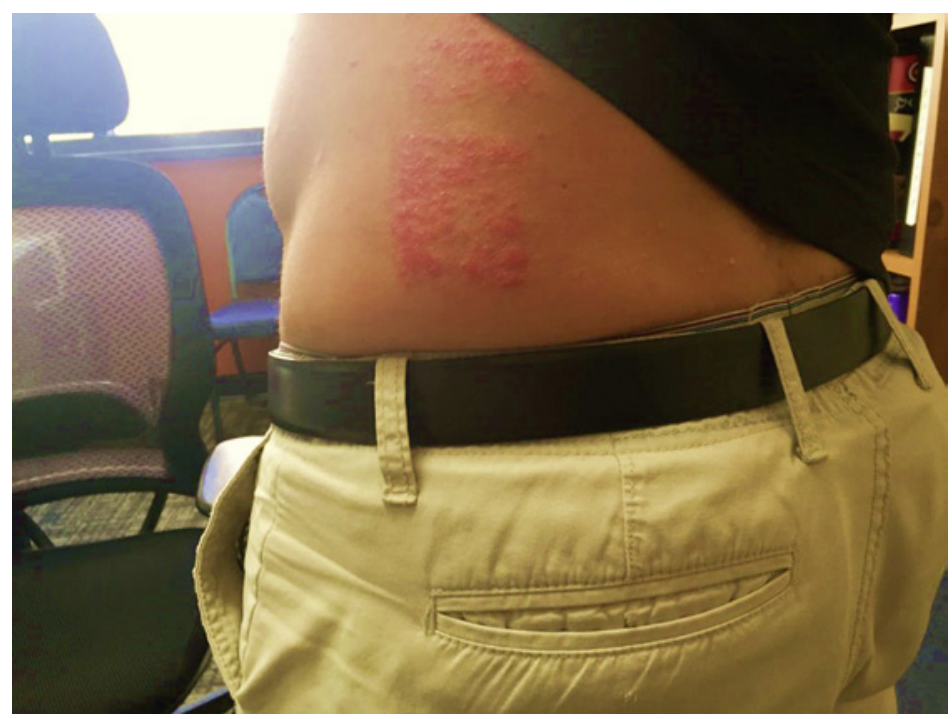

Fig. 2. Psoriasis lesions distant from patch application point.

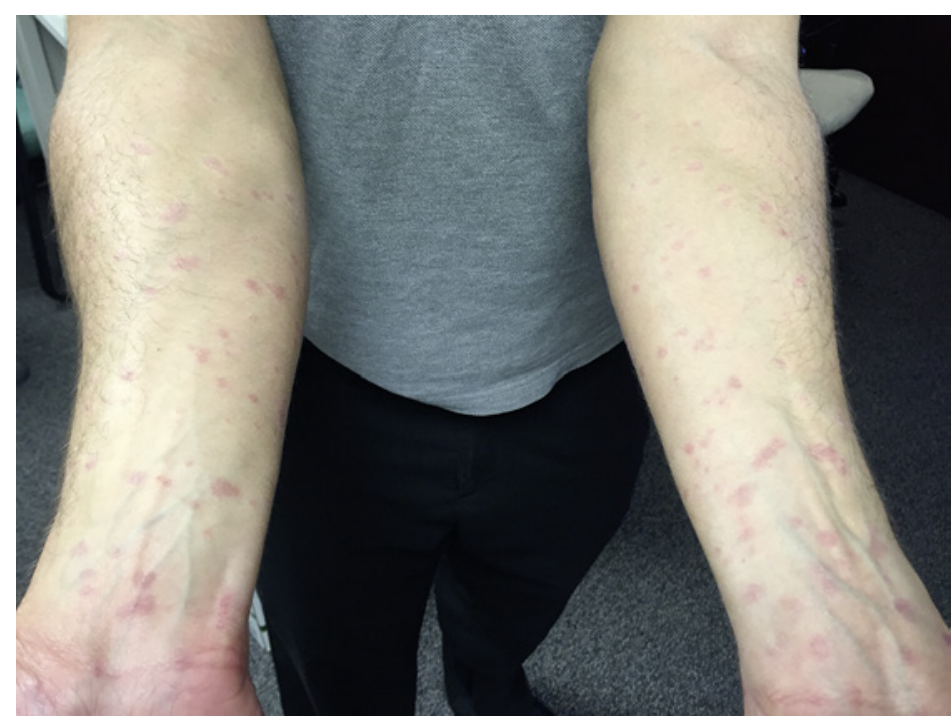

sponding history for diagnosis; this case demonstrates the challenges of relying on history alone for final identification.

A 35-year-old Caucasian male presented to Allergy clinic for evaluation of a new rash on his flank. Four days prior to the development of the rash, the patient began a smoking cessation program utilizing transdermal nicotine patches. After 2 days, a rash was noted and they were discontinued. The rash at presentation was a well-demarcated, erythematous, papular patch measuring approximately $20 \mathrm{~cm} \times 10 \mathrm{~cm}$ (Fig. 1). He also reported that he was being treated for a presumed Group A Streptococcus throat infection diagnosed 1 week prior.

Initially felt to be an allergic $\mathrm{CD}$ to the nicotine patches, he was prescribed a mediumpotency topical steroid. Despite this, the rash worsened and began to involve other areas of skin as well (Fig. 2). Patch testing on unaffected skin was negative to three standard panels of epicutaneous patch testing and a new nicotine patch. Additionally, biopsies of the original rash and one distal from the original eruption showed "psoriasiform and superficial perivascular dermatitis with mounds of parakeratosis and neutrophils," consistent with guttate 
psoriasis (data not shown). The patient was treated with UV light and high-potency topical steroids with improvement, though he continues to have flares occasionally.

Psoriasis is a chronic immune-mediated skin condition resulting in well-demarcated erythematous plaque formation. Multiple presentations of psoriasis exist, including plaque, guttate, pustular, and erythrodermic forms. Along with genetic risk factors, environmental factors are known to trigger or exacerbate psoriasis, including trauma, infections, and medications [1]. Specifically, streptococcal infections are known to be inducers of guttate psoriasis. The mechanism for the induction of psoriasis by infection is hypothesized to be due to superantigen-induced T cells which produce increased amounts of interferon- $\gamma$ locally [2].

Transdermal nicotine patches have been implicated in causing local irritation [3] and CD [4]. Nicotine's potent vasodilatory properties have been implicated in the etiology of skin irritation; methacrylates, a nicotine patch component, can induce CD as well $[5,6]$.

Early reports hypothesized that CD and psoriasis could not occur simultaneously due to their seemingly contradictory mechanisms of action: CD causes increased cell apoptosis, in stark contrast to the poor cell turnover seen in psoriasis $[7,8]$. Newer studies have shown that $\mathrm{CD}$ and psoriasis do exist together and CD may induce psoriasis through an isomorphic response as well as through the contact allergen itself $[9,10]$. Other provoking factors include physical trauma, burns, friction, insect bites, surgical incision, radiation exposure, medications, needle acupuncture, and tattoos [11]. Finally, the risk of CD in patients with psoriasis may be allergen-specific and often will appear later due to the delay of migration of antigenpresenting cells in psoriasis patients $[8,12]$.

CD relies heavily on clinical history for diagnosis; this case demonstrates that history can be misleading. The time of onset and the distribution of the rash was highly concerning for CD. However, after poor response to traditional CD therapies, and without confirmatory findings on patch testing, the biopsy ultimately confirmed the diagnosis of psoriasis. In retrospect, it is likely that the preceding presumed Streptococcal infection was the primary inducer of guttate psoriasis and the concomitant friction irritation of the transdermal patch was a secondary point of inflammatory response.

\section{Statement of Ethics}

Patient consent was obtained and granted. This report does not include any animal experiments.

\section{Disclosure Statement}

The authors have no conflicts of interests to declare.

\section{Funding Sources}

No funding was received.

\section{Author Contributions}

Both authors were extensively involved in data collection, research, and writing of the manuscript. 


\section{References}

1 Greb JE, Goldminz AM, Elder JT, et al. Psoriasis. Natl Rev. 2016;(2):1-17.

2 Fry L, Baker BS. Triggering psoriasis: the role of infections and medications. Clin Dermatol. 2007 Nov-Dec; 25(6):606-15.

3 Greenland S, Satterfield MH, Lanes SF. A meta-analysis to assess the incidence of adverse effects associated with the transdermal nicotine patch. Drug Saf. 1998 Apr;18(4):297-308.

4 Bircher AJ, Howald H, Rufli T. Adverse skin reactions to nicotine in a transdermal therapeutic system. Contact Dermat. 1991 Oct;25(4):230-6.

5 von Bahr B, Wahlberg JE. Reactivity to nicotine patches wrongly blamed on contact allergy. Contact Dermat. 1997 Jul;37(1):44-5.

6 Dwyer CM, Forsyth A. Allergic contact dermatitis from methacrylates in a nicotine transdermal patch. Contact Dermat. 1994 May;30(5):309-10.

7 Henseler T, Christophers E. Disease concomitance in psoriasis. J Am Acad Dermatol. 1995 Jun;32(6):982-6.

8 Quaranta M, Eyerich S, Knapp B, Nasorri F, Scarponi C, Mattii M, et al. Allergic contact dermatitis in psoriasis patients: typical, delayed, and non-interacting. PLoS One. 2014 Jul;9(7):e101814.

9 Weiss G, Shemer A, Trau H. The Koebner phenomenon: review of the literature. J Eur Acad Dermatol Venereol. 2002 May;16(3):241-8.

10 Sagi L, Trau H. The Koebner phenomenon. Clin Dermatol. 2011 Mar-Apr;29(2):231-6.

11 Orzan OA, Popa LG, Vexler ES, et al. Tattoo-induced psoriasis. J Med Life. 2014;7 Spec No. 2:65-8.

12 Claßen A, Buhl T, Schubert S, et al. The frequency of specific contact allergies is reduced in patients with psoriasis. Br J Dermatol. 2019 Feb; 180(2):315-20. 